\title{
Rupture-Associated Changes of Cerebral Aneurysm Geometry: High-Resolution 3D Imaging before and after Rupture
}

\author{
J.J. Schneiders, H.A. Marquering, R. van den Berg, E. VanBavel, B. Velthuis, G.J.E. Rinkel, and C.B. Majoie
}

\begin{abstract}
BACKGROUND AND PURPOSE: Comparisons of geometric data of ruptured and unruptured aneurysms may yield risk factors for rupture. Data on changes of geometric measures associated with rupture are, however, sparse, because patients with ruptured aneurysms rarely have undergone previous imaging of the intracranial vasculature. We had the opportunity to assess 3D geometric differences of aneurysms before and after rupture. The purpose of this study was to evaluate possible differences between prerupture and postrupture imaging of a ruptured intracranial aneurysm.
\end{abstract}

MATERIALS AND METHODS: Using high-quality 3D image data, we generated 3D geometric models before and after rupture and compared these for changes in aneurysm volume and displacement. A neuroradiologist qualitatively assessed aneurysm shape change, the presence of perianeurysmal hematoma, and subsequent mass effect exerted on aneurysm and parent vessels.

RESULTS: Aneurysm volume was larger in the postrupture imaging in 7 of 9 aneurysms, with a median increase of $38 \%$ and an average increase of $137 \%$. Three aneurysms had new lobulations on postrupture imaging; 2 other aneurysms were displaced up to $5 \mathrm{~mm}$ and had changed in geometry due to perianeurysmal hematoma.

CONCLUSIONS: Geometric comparisons of aneurysms before and after rupture show a large volume increase, origination of lobulations, and displacement due to perianeurysmal hematoma. Geometric and hemodynamic comparison of series of unruptured and ruptured aneurysms in the search for rupture-risk-related factors should be interpreted with caution.

U

nruptured intracranial aneurysms are found in approximately $3 \%$ of the population. ${ }^{1}$ Once they are detected, the decision for preventive treatment has to be weighed against the risk of rupture, with inherent high case fatality and morbidity. ${ }^{2}$ Prediction of rupture of intracranial aneurysms remains poor, with size as the most important risk factor. However, not all large aneurysms would rupture if left untreated; whereas small aneurysms, which are often left untreated, do sometimes rupture during follow-up. Better predictors

\footnotetext{
Received October 12, 2013; accepted after revision December 5.

From the Departments of Radiology (J.J.S., H.A.M., R.v.d.B., C.B.M.) and Biomedical Engineering and Physics (H.A.M., E.V.), Academic Medical Center, Amsterdam, the Netherlands; and Department of Neurology and Neurosurgery (G.J.E.R.), Brain Center Rudolf Magnus, and Department of Radiology (B.V.), University Medical Center Utrecht, Utrecht, the Netherlands.

J.J. Schneiders and H.A. Marquering have contributed equally to this article.

This work was supported by a grant from the Nuts-OHRA Foundation, Amsterdam, the Netherlands.

Please address correspondence to Joppe Schneiders, MD, Department of Radiology, Cl, Academic Medical Center, University of Amsterdam, PO Box 22660, 1100 DD Amsterdam, the Netherlands: e-mail: j.j.schneiders@amc.uva.nl

三 Indicates article with supplemental on-line table.

http://dx.doi.org/10.3174/ajnr.A3866
}

are, therefore, needed. Intra-aneurysmal hemodynamic characteristics may have predictive value. ${ }^{3,4}$

Computational fluid dynamics have been applied to simulate hemodynamic flow patterns in the aneurysm and surrounding vessels to relate hemodynamic characteristics with aneurysmal rupture risk. ${ }^{4,5}$ Several studies indeed found differences in flow patterns between ruptured and unruptured aneurysms. ${ }^{3,4,6}$ However, if one compares ruptured with unruptured aneurysms, the potential changes of the aneurysm geometry before, during, or after rupture itself are neglected.

The rupture of the aneurysm may result in shape changes of the aneurysm due to changes in the aneurysmal sac. These changes potentially alter the aneurysmal or perianeurysmal geometry and its related hemodynamic patterns. It is, therefore, pivotal to know whether changes before, during, or shortly after rupture of aneurysms in themselves affect aneurysm geometry and aneurysm hemodynamics; and if so, what these changes are. Such data are difficult to collect because high-quality images of intracranial aneurysms before and after rupture are rare. We had the opportunity to assess changes in aneurysm geometry associated with rupture in a series of 9 patients by using advanced image registration of high-quality $3 \mathrm{D}$ imaging data performed before and after rupture. 
Table 1: Imaging modalities used before and after rupture

\begin{tabular}{llccc}
\hline No. & $\begin{array}{c}\text { Imaging Modality } \\
\text { before Rupture }\end{array}$ & Resolution $(\mathbf{m m})$ & $\begin{array}{c}\text { Imaging Modality } \\
\text { after Rupture }\end{array}$ & Resolution $(\mathbf{m m})$ \\
\hline 1 & 3D TOF MRA & $0.49 \times 0.49 \times 1.2$ & 3D RA & $0.22 \times 0.22 \times 0.22$ \\
2 & 3D TOF MRA & $0.31 \times 0.31 \times 1.0$ & CTA & $0.31 \times 0.31 \times 0.45$ \\
3 & CTA & $0.33 \times 0.33 \times 1.0$ & CTA & $0.35 \times 0.35 \times 0.33$ \\
4 & 3D PC MRA & $0.78 \times 0.78 \times 1.0$ & 3D RA & $0.25 \times 0.25 \times 0.25$ \\
5 & 3D TOF MRA & $0.45 \times 0.45 \times 1.0$ & 3D RA & $0.39 \times 0.39 \times 0.39$ \\
6 & CTA & $0.43 \times 0.43 \times 0.9$ & 3D RA & $0.22 \times 0.22 \times 0.22$ \\
7 & 3D TOF MRA & $0.20 \times 0.20 \times 1.0$ & 3D RA & $0.17 \times 0.17 \times 0.17$ \\
8 & 3D TOF MRA & $0.35 \times 0.35 \times 1.4$ & 3D RA & $0.17 \times 0.17 \times 0.17$ \\
9 & CTA & $0.33 \times 0.33 \times 1.3$ & 3D RA & $0.09 \times 0.09 \times 0.09$ \\
\hline
\end{tabular}

Note:-PC indicates phase-contrast; RA, rotational angiography

\section{MATERIALS AND METHODS}

We searched the neurovascular data bases for patients presenting with aneurysmal subarachnoid hemorrhage who underwent 3D cerebral angiography (3D rotational angiography, MRA, or CTA) before the aneurysm rupture. We retrieved imaging of 9 patients from 2 institutions (Academic Medical Center [Amsterdam] and University Medical Center Utrecht) collected during a 6-year period. Approval of the medical ethics committee was given for this retrospective analysis of anonymized patient data. Informed consent was waived because no diagnostic tests other than routine clinical imaging were used in this study.

A number of geometric features of the intracranial aneurysms were quantitatively and qualitatively determined in both pre- and postrupture imaging. The quantitatively determined features were aneurysm volume and maximal aneurysm diameter. The maximal diameter was defined as the maximal distance between 2 locations on the aneurysm surface with their connecting line completely located in the aneurysm sac. Furthermore, we determined displacement and shape changes between the 2 image datasets. To better assess shape change, we registered the pre- and postrupture imaging data so that the aneurysm sac was optimally matched. To determine the displacement of the aneurysm, we registered a proximal segment of the parent artery of the pre- and postrupture imaging data.

\section{Registration}

The pre- and postrupture images were registered by a closedsource in-house developed software system for modality registration and visualization (WorldMatch). ${ }^{7}$ The registration of the images was performed twice: First, the images were registered by using a volume of interest around the aneurysm sac to obtain an optimal match of the aneurysm itself. Second, the images were registered to obtain an optimal match of the feeding artery. A single observer (H.A.M.), who had no clinical information other than the location of the aneurysm, performed these registrations. The highest resolution of the 2 image datasets was used for the spatial grid of the registered images.

\section{Segmentation}

The aneurysms and adjacent arteries were segmented by using level set segmentation (Vascular Modeling ToolKit; http:// www.vmtk.org). ${ }^{8}$ An experienced neuroradiologist (C.B.M.) inspected all segmentations for accuracy by using all available image data. Segmentation inaccuracies were manually corrected by using ITK-SNAP, a software application for medical image segmen- tation and manual delineation (ITKSNAP 2.2.0, http://www.itksnap.org), ${ }^{9}$ until the neuroradiologist decided the segmentation was accurate. Subsequently, the aneurysm sac was separated from the arterial tree by using ITKSNAP. These aneurysm sac models were used to calculate the volume of the aneurysms and aneurysm shape changes.

Surface models were generated from the matched segmentations. The registration was inspected by a second observer (R.v.d.B.) and adjusted if needed by using a combination of iterative closest point registration and manual transforms.

\section{Quantitative Measurements}

The volume of the aneurysms was defined as the number of segmented aneurysm voxels multiplied by the volume of a single voxel. Absolute and relative volume changes were determined. We defined the displacement of the aneurysm sac as the distance between the center of the pre- and postrupture aneurysm sacs, by using the images in which the parent arteries were registered. The center of the aneurysm was mathematically defined as the center of the mass of the segmented aneurysm volume.

The shape change was quantified by using the point-surface distances of the surface of the pre- and postrupture aneurysm segmentations in images in which the aneurysm domes were registered. A point-surface distance is the minimal distance of a location of one aneurysm surface to the surface of the other. The average and maximum point-surface distances are presented in the On-line Table. To avoid overestimations due to modalitydriven variations in the definition of the aneurysm neck, ${ }^{10}$ we ensured that this maximum distance was remote from the neck.

\section{Qualitative Assessments}

The neuroradiologist (C.B.M.) evaluated all the available pre- and postrupture imaging for the presence of intra-aneurysmal thrombus that could influence angiography-derived segmentations. The postrupture data were also examined for the presence of a perianeurysmal hematoma and new lobulations. In the presence of perianeurysmal hematoma, it was judged whether this mass had induced a displacement or shape change in the aneurysm.

The postrupture DSA examinations were also assessed for the presence of new lobulations and pseudoaneurysms (a contained extraluminal rupture within a reorganized clot). Pseudoaneurysms were differentiated from true lobulations by their appearance with delayed and incomplete opacification and delayed washout. $^{11}$

\section{RESULTS}

3D angiographic imaging, before and after rupture, was available for 9 patients. The image modalities used and the resolution are shown in Table 1 . The average time between prerupture imaging and rupture was 1 year 127 days, with an SD of 1 year 199 days. The On-line Table shows the geometric measures of the aneurysms pre- and postrupture. Seven aneurysms showed an increase 


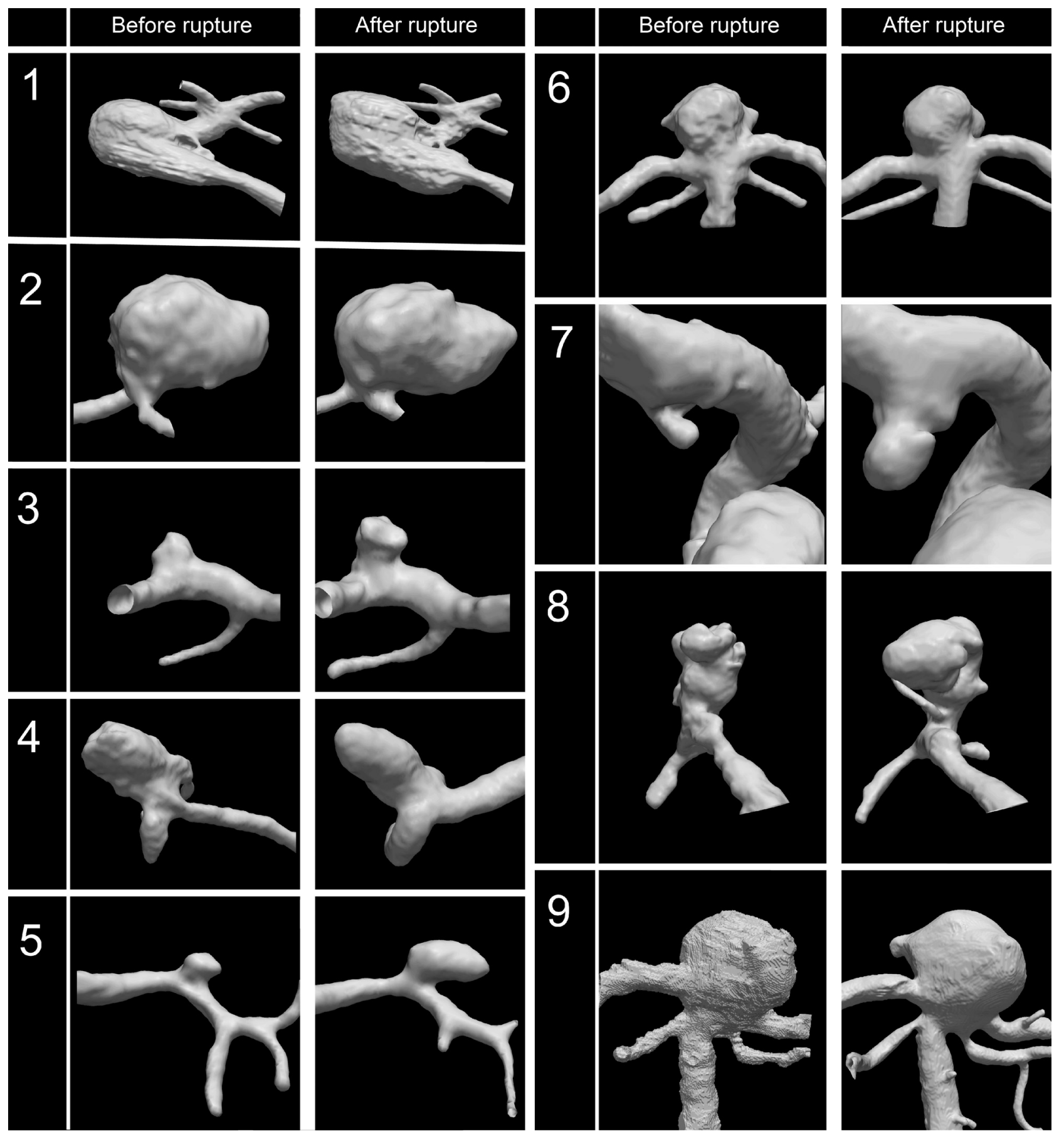

FIG 1. Volume rendering of angiographic imaging data before and after rupture for all cases. Cases 1 and 2 show the largest absolute increase in aneurysm volume $\left(+433\right.$ and $+613 \mathrm{~mm}^{3}$, respectively); case 5 and 7 , the largest relative increase in aneurysm volume (176\% and $832 \%$, respectively).

in volume with a median increase of $38 \%$ and an average increase of $137 \pm 266 \%$.

Two aneurysms (cases 1 and 2 in Fig 1) showed a large absolute volume increase of 433 and $613 \mathrm{~mm}^{3}$, respectively, in the postrupture imaging. This increase had occurred in a relatively short time of 183 and 72 days, respectively. Furthermore, in case 7, a large relative volume increase of $832 \%$ had occurred within a time frame of no more than 14 days (Fig 2).

Table 2 shows the qualitative observations made by the neuroradiologist. Three aneurysms had new lobulations on postrupture im- aging; no pseudoaneurysms and no intra-aneurysmal thrombus were found in any aneurysm. A perianeurysmal hematoma was present in 3 cases, 2 of which exerted mass effect on the aneurysm, thereby changing the aneurysm geometry (see Fig 3 for an example).

\section{DISCUSSION}

On the basis of pre- and postrupture 3D imaging of ruptured aneurysms, we found significant volume changes, new lobulations, and aneurysm displacement due to hematoma mass effect after rupture. 
Most aneurysms showed an increase in volume in postrupture imaging. It has been shown that aneurysm growth is associated with an increased rupture risk. ${ }^{12}$ Annual growth in untreated unruptured aneurysms is reported to be between $1.5 \%$ and $22.7 \%$, corresponding to a diameter growth between 0.12 and $1.3 \mathrm{~mm} .{ }^{13-15} \mathrm{In}$ the current study, the average growth rate was much larger $(4.8 \mathrm{~mm} /$ year). Our findings are more in line with another study that included 13 ruptured aneurysms with pre- and postrupture 2D-DSA, which showed an average increase in maximal diameter of $3.2 \mathrm{~mm} /$ year. ${ }^{16} \mathrm{~A}$ possible explanation of the difference in growth rate compared with previous imaging follow-up studies of unruptured aneurysms ${ }^{13-15}$ is that the population of Rahman et $\mathrm{al}^{16}$ and our study consisted of selected ruptured aneurysms, in which the rupture itself may be associated with larger growth.

We found no apparent examples of aneurysm shrinkage after rupture. ${ }^{16,17}$ In 2 instances, we found a small volume decrease of $3 \%$ and $4 \%$, but these changes fall within the margin of error of the volume measurement due to differences in the modalities used and/or processing methods.

Because of the relatively large time interval between prerupture and postrupture imaging, no conclusions on the causality or the timing of these changes can be drawn. The observed changes could have occurred before, during, or shortly after rupture. Hence, we can only conclude that they were associated with rupture. However, in case of a large hematoma formation, an obvious causal relation exists between rupture and consequent geometry change because large hematomas can displace and deform the aneurysm.

A few aneurysms were displaced due to hematoma mass effect, thereby changing the geometric relation with their supplying artery. The resulting displacement may have an effect on the relative position of the aneurysm parent vessel and location of inflow. In our cases, the displacement changed the angle of the parent vessel with regard to the aneurysm neck. The parent vessel angle has been described as a surrogate measure for the direction of the blood flow into the aneurysm and was proposed as a risk factor for aneurysm rupture. ${ }^{18,19}$ The supplying artery is also an important determinant for intra-aneurysmal hemodynamics calculated by using computational fluid dynamics. The

FIG 2. Case 7. Lateral view of 2D DSA $(A)$ and 3DRA $(B)$ before rupture shows a small aneurysm at the posterior communicating artery. Postrupture 2D DSA $(C)$ and 3DRA $(D)$ show a large increase of aneurysm volume and a new lobulation at the aneurysm dome.

\section{Table 2: Qualitative assessment of pre- and postrupture imaging}

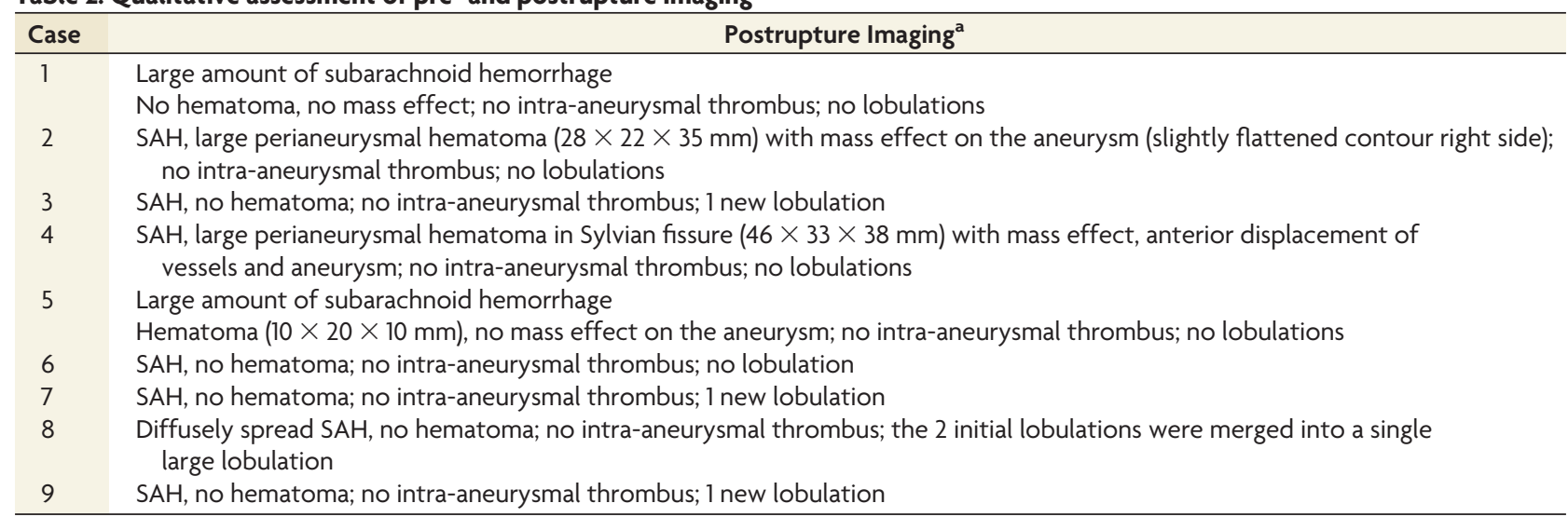

${ }^{a}$ None of the prerupture imaging showed an intra-aneurysmal thrombus. Only case 8 showed lobulations on the prerupture imaging (2 lobulations). 

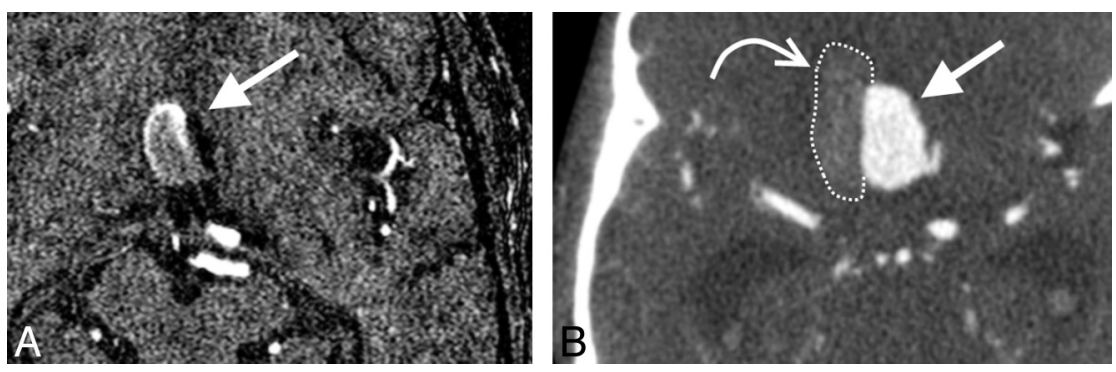

FIG 3. Case 2. A, Axial 3D TOF MRA source image shows an anterior communicating artery aneurysm (arrow). B, Axial CTA source image after rupture shows an increase of aneurysm volume and flattening of the right aneurysm border and displacement of the aneurysm to the left side due to a large perianeurysmal hematoma (curved arrow).

change in geometry of the parent artery and aneurysm sac may have important implications for measurements of intra-aneurysmal hemodynamics by using computational fluid dynamics. Computational fluid dynamics-based calculations are commonly used to estimate hemodynamic risk factors, whereas the postrupture geometry is assumed representative of the geometry before rupture. This assumption may not hold for the change in parent artery-aneurysm geometry shown here.

Some aneurysms showed a new focal bulging or lobulation that was not present on prerupture imaging Although lobulations may have been caused by the rupture itself, the high number of lobulations are in line with previous studies in which the presence of lobulations was associated with an increased rupture risk. ${ }^{19,20}$ One may argue that some of these new lobulations could also be pseudoaneurysms (ie, a contained extraluminal rupture within a reorganized clot). We think this is unlikely because on postrupture DSA examinations, they did not demonstrate delayed opacification and washout. ${ }^{11}$

A limitation of this study is that we compared images and segmentations originating from different imaging modalities. Differences in the segmented volumes may be caused by differences in these modalities. Especially 3D TOF MRA is prone to introduce angiographic inaccuracies due to signal loss and may underestimate aneurysm volume. As a result, volume comparison of prerupture MRA with postrupture 3D rotational angiography or CTA could incorrectly be interpreted as aneurysm growth. Although this effect could produce incorrect conclusions on aneurysm growth, the increase in size in some cases in this study was much too large to be explained by this limitation of MRA imaging.

\section{CONCLUSIONS}

The geometric changes we found in aneurysms before and after rupture show that direct comparison of these geometric and hemodynamic features between series of ruptured and unruptured aneurysms for assessment of risk factors should be considered with caution.

Disclosures: Joppe J. Schneiders—RELATED: supported by the NUTS-OHRA Foundation (grant SNO-T-0702-92). Brigitta Velthuis-UNRELATED: Payment for Lectures (including service on Speakers Bureaus): Philips Healthcare.* Charles B. MajoieRELATED: Grant: NUTS-OHRA Foundation, * UNRELATED: Grants/Grants Pending: Dutch Heart Foundation. * Money paid to the institution.

\section{REFERENCES}

1. Vlak MH, Algra A, Brandenburg R, et al. Prevalence of unruptured intracranial aneurysms, with emphasis on sex, age, comorbidity, country, and time period: a systematic review and meta-analysis. Lancet Neurol 2011;10:626-36
2. McDonald JS, McDonald RJ, Fan J, et al. Comparative effectiveness of ruptured cerebral aneurysm therapies: propensity score analysis of clipping versus coiling. AJNR Am J Neuroradiol 2014;35:164-69

3. Cebral JR, Mut F, Weir J, et al. Association of hemodynamic characteristics and cerebral aneurysm rupture. AJNR Am J Neuroradiol 2011;32:264-70

4. Xiang J, Natarajan SK, Tremmel M, et al. Hemodynamic-morphologic discriminants for intracranial aneurysm rupture. Stroke 2011;42:144-52

5. Cebral JR, Castro MA, Burgess JE, et al. Characterization of cerebral aneurysms for assessing risk of rupture by using patient-specific computational hemodynamics models. AJNR Am J Neuroradiol 2005; 26:2550-59

6. Jou LD, Lee DH, Morsi $\mathrm{H}$, et al. Wall shear stress on ruptured and unruptured intracranial aneurysms at the internal carotid artery. AJNR Am J Neuroradiol 2008;29:1761-67

7. Wolthaus JW, van Herk M, Muller SH, et al. Fusion of respirationcorrelated PET and CT scans: correlated lung tumour motion in anatomical and functional scans. Phys Med Biol 2005;50:1569-83

8. Antiga L, Piccinelli M, Botti L, et al. An image-based modeling framework for patient-specific computational hemodynamics. Med Biol Eng Comput 2008;46:1097-112

9. Yushkevich P, Piven J, Hazlett HC, et al. User-guided 3D active contour segmentation of anatomical structures: significantly improved efficiency and reliability. Neuroimage 2006;31:1116-28

10. Schneiders JJ, Marquering HA, Antiga L, et al. Intracranial aneurysm neck size overestimation with $3 \mathrm{D}$ rotational angiography: the impact on intra-aneurysmal hemodynamics simulated with computational fluid dynamics. AJNR Am J Neuroradiol 2013;34:121-28

11. D’Urso PI, Loumiotis I, Milligan BD, et al. "Real time” angiographic evidence of "pseudoaneurysm" formation after aneurysm rebleeding. Neurocrit Care 2011;14:459-62

12. Villablanca JP, Duckwiler GR, Jahan R, et al. Natural history of asymptomatic unruptured cerebral aneurysms evaluated at CT angiography: growth and rupture incidence and correlation with epidemiologic risk factors. Radiology 2013;269:258-65

13. Wermer MJ, van der Schaaf IC, Velthuis BK, et al. Yield of short-term follow-up CT/MR angiography for small aneurysms detected at screening. Stroke 2006;37:414-18

14. Juvela S, Porras M, Poussa K. Natural history of unruptured intracranial aneurysms: probability of and risk factors for aneurysm rupture. J Neurosurg 2008;108:1052-60

15. Ferns SP, Sprengers ME, van Rooij WJ, et al. De novo aneurysm formation and growth of untreated aneurysms: a 5-year MRA follow-up in a large cohort of patients with coiled aneurysms and review of the literature. Stroke 2011;42:313-18

16. Rahman M, Ogilvy CS, Zipfel GJ, et al. Unruptured cerebral aneurysms do not shrink when they rupture: multicenter collaborative aneurysm study group. Neurosurgery 2011;68:155-60, discussion 160-61

17. Kataoka K, Taneda M, Asai T, et al. Difference in nature of ruptured and unruptured cerebral aneurysms. Lancet 2000;355:203

18. Baharoglu MI, Schirmer CM, Hoit DA, et al. Aneurysm inflow-angle as a discriminant for rupture in sidewall cerebral aneurysms: morphometric and computational fluid dynamic analysis. Stroke 2010;41:1423-30

19. de Rooij NK, Velthuis BK, Algra A, et al. Configuration of the circle of Willis, direction of flow, and shape of the aneurysm as risk factors for rupture of intracranial aneurysms. J Neurol 2009;256:45-50

20. Beck J, Rohde S, el Beltagy M, et al. Difference in configuration of ruptured and unruptured intracranial aneurysms determined by biplanar digital subtraction angiography. Acta Neurochir (Wien) 2003;145:861-65, discussion 865 